\title{
Analysis of Industrial Matching Capacity in Qingdao Blue Economic Zone
}

\author{
Zhiyao Zhang \\ Qingdao University of Science \& Technology, Qingdao 266061 China \\ qustzzy@qust.edu.cn
}

Keywords: Blue economy; Industrial matching; Support system; Countermeasures

\begin{abstract}
This paper discusses the connotation of the blue economic industry matching, the supporting system of industrial matching, the dynamic change rule of industrial matching, analyzes the current situation of industrial matching in Qingdao Blue Economic Zone. The countermeasures and suggestions are put forward to improve the industrial matching of the Blue Economic Zone in Qingdao.

The practice of economic development has proved that behind the advanced industries of a certain region, there must be a developed supporting system. The improvement of industry supporting level will produce strong spillover effect, and it will play an important role in the transformation of the industrial structure and the competitive ability of the leading enterprises." The development plan of the blue economic zone of the Shandong peninsula" was approved by the State Council, indicating that the construction of the Blue Economic Zone in the Shandong Peninsula has been formally upgraded to a national strategy ${ }^{[1]}$. Improving the industry matching of Qingdao Blue Economic Zone, it will form a virtuous circle of "industry supporting promotion $\rightarrow$ attracting investment $\rightarrow$ industrial aggregation $\rightarrow$ matching optimization of industry", and is of critical value to the success or failure of the blue economy.
\end{abstract}

\section{The Connotations of Blue Economy and Industry}

The blue economy is the sum of the economic activities that directly develop ocean, utilize ocean, ocean protect and rely on the ocean. It can be extended to the marine economy, the coastal economy, the sea economy and the overseas economy. It is a new development thinking under the concept of sustainable development of the integration of the past marine economy and green economic development. Different from the traditional marine economy, the greatest characteristics of the Blue Economic Zone, is the coordinated development of land and sea and the coordinated development of economy, society and ecology ${ }^{[2]}$. The blue economic zone is the concept of sea related space, is also an innovation area. Based on economy, science and technology, society and open of sea-land integration area and system innovation system ${ }^{[3]}$. The blue economic zone is the concept of sea related space, is also an innovation area, is a land and sea integration area and system innovation system based on economy, science and technology, society and opening to the outside world. The blue economic zone is a modern marine characteristic economic zone to coordinate development of economy and ecology and society, is taking the construction of marine ecological civilization as the principal principle, taking the land and sea as a fundamental way to rationally configure the marine advantageous industry, the coastal industry and the sea industry.

Industry matching is the result of specialization of industrial division of labor, generally divided into industrial supporting and inter industry matching. Narrow industry matching is based on the whole production process and chain links, and the related production areas or sectors are matched with the production process and technology links. The broad sense of industrial matching is the comprehensive support capability of providing support technology elements and social service elements for industrial development. It includes not only the narrow sense of the industry, but also the social service system, such as the market, the amount, the human resources, and so on ${ }^{[4],[10]}$. The types of industry matching can be divided into four types: market transaction matching, order matching, strategic alliance supporting, equity alliances, supporting enterprises, internal supporting 
businesses, etc.

Blue economic industry matching is a comprehensive system of supporting elements, which involves the upstream and downstream related industries with inherent links in the process of production, operation and sales of enterprises. It also involves related product promotion, resource integration, service, human resources, technical resources, consumer market development, etc.

\section{Supporting System of Blue Economic Industry}

The development of blue ocean industry needs the support of the supporting industrial system, and it is an important factor to attract investment. A good industry supporting system can effectively reduce the risk and comprehensive cost of enterprise management ${ }^{[6]}$. Under the industrial supporting system, uncertainty risk control and comprehensive cost factor of enterprise survival environment are the core problems of enterprise survival and development.

The supporting industry system of blue ocean industry includes not only the purchase of related parts and services, but also the support of related infrastructure and social service system. For example, the manufacturing industry of marine equipment involves dozens of related industries, such as machinery manufacturing, electrical manufacturing, rubber plastics and other related industries, and is closely related to technology support, talent training, financial services and other related businesses. The development of coastal tourism needs not only the cooperation of catering, accommodation, business, finance and other service sectors, but also the support from many sectors such as municipal construction, transportation and other industries (See Figure 1).

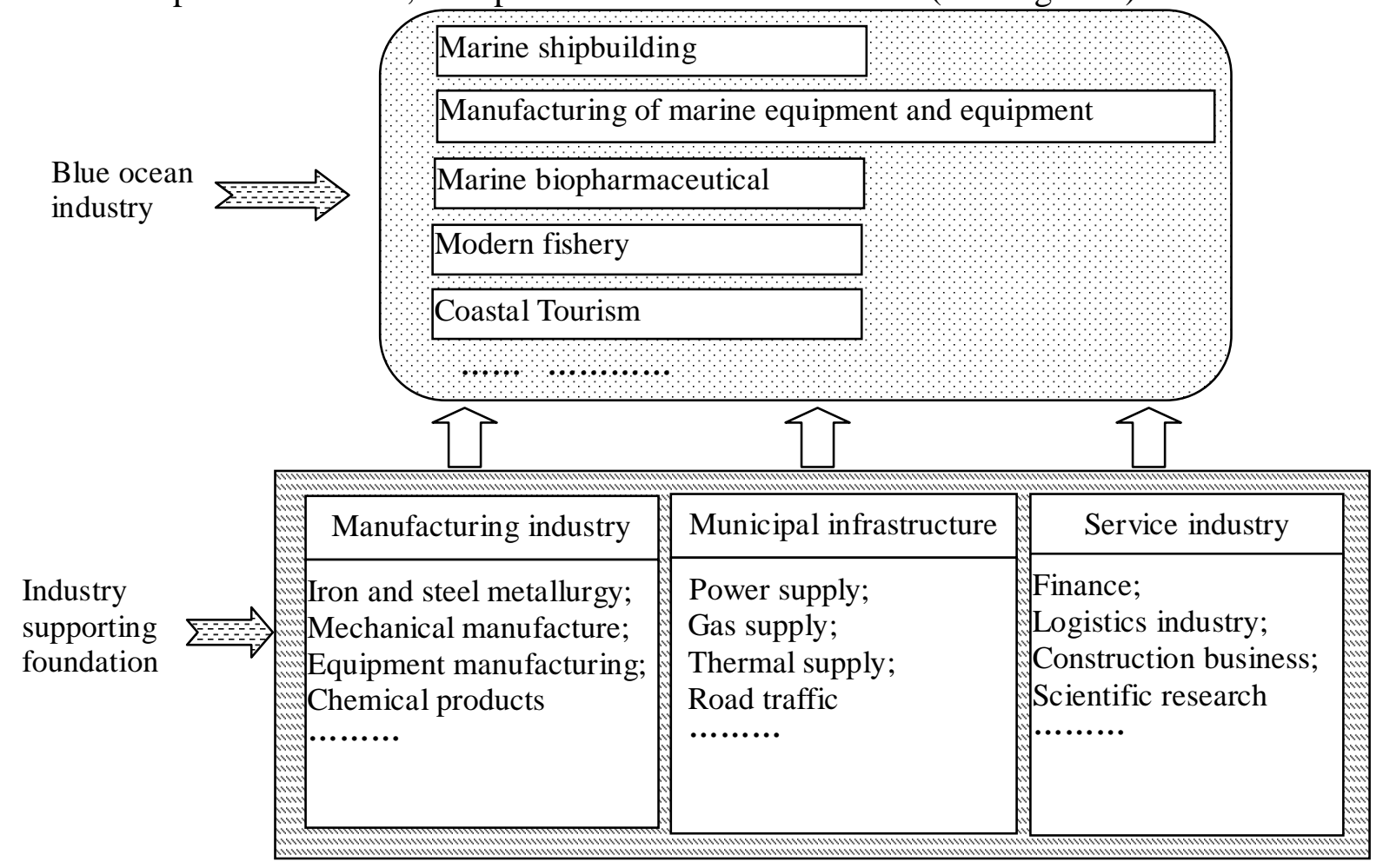

Figure 1. The supporting structure of the blue economy industry

Industrial support is based on a relatively complete industrial base and industrial scale. Without a relatively complete industrial economic system and a certain industrial scale, it is impossible to achieve a better industry. Under the environment of market economy, enterprises purchase parts and service according to the rules of market economy to achieve. Only when the local related industries have competitive advantage in quality, price and service, can the local purchasing behavior be realized, and then complete the supporting system of the local industry chain. Otherwise, the enterprises are purchasing from other places in accordance with the rules of cost control. Too much foreign procurement leads to the extension of the supply chain, the increase of cost, and the reduction of efficiency, which will affect the comprehensive competitiveness. 


\section{The Dynamic Changes of the Blue Economy Industry}

The Dynamic Mechanism of the Blue Economy Industry. Industrial matching is a dynamic process, and the local cooperative relationship formed between enterprises has changed with the development of technology ${ }^{[7]}$. With the improvement of local industrial technology, from outside of the region purchase of supporting components or services can achieve local purchase. At the same time, the relationship between supply and demand has been formed at present, due to the upgrading of leading products, if the technology of supporting related industries is not synchronized, the old supporting relationship will be broken, leading enterprises will inevitably choose external matching (See Figure 2).

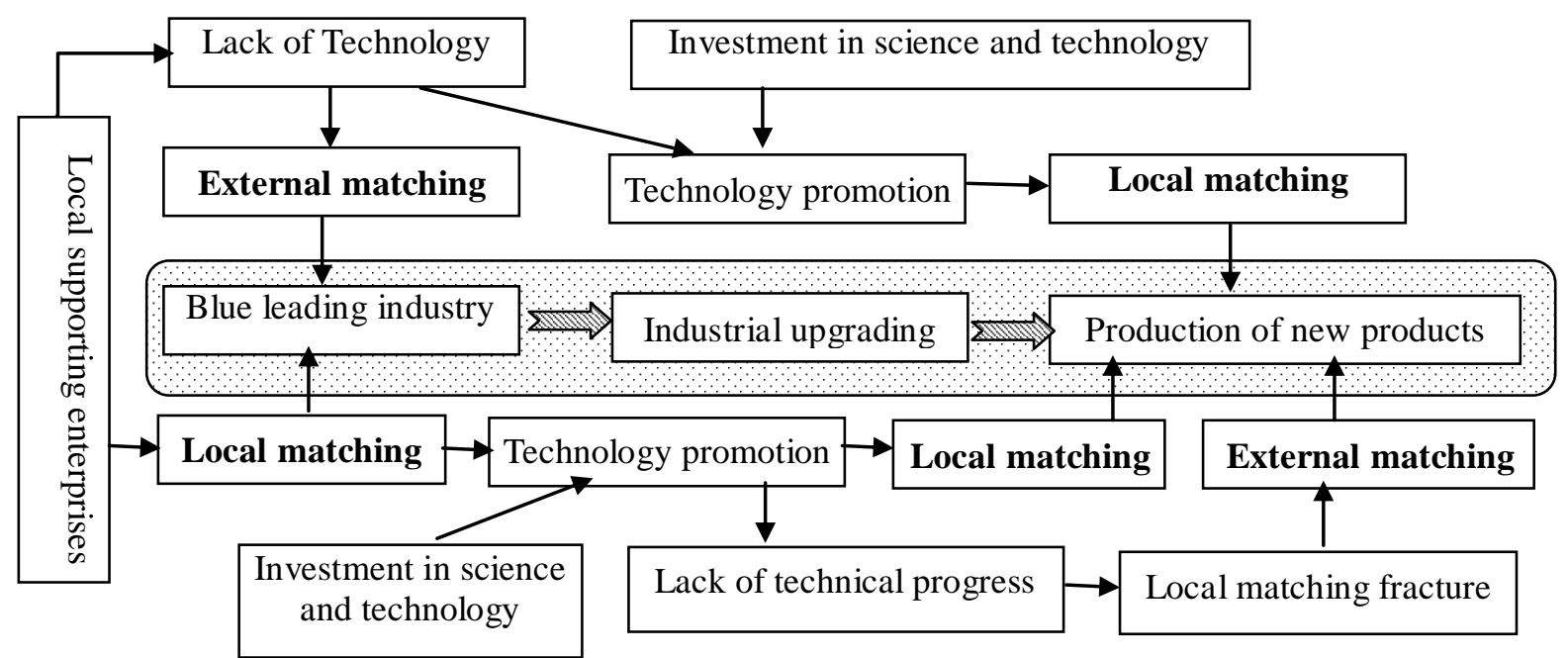

Figure 2. Dynamic changes in industrial matching in Blue Economic Zone

Industrial Measurement Index in Blue Economic Zone. The local supporting capacity of industrial development in blue economic zone depends on three factors. One is to have a certain scale of industrial base. The two is that the supporting industry should have the competitive strength. Only the competitive parts or services can achieve the local support successfully. The three is that the supporting industry should have the potential of technological progress. (see Figure 3).

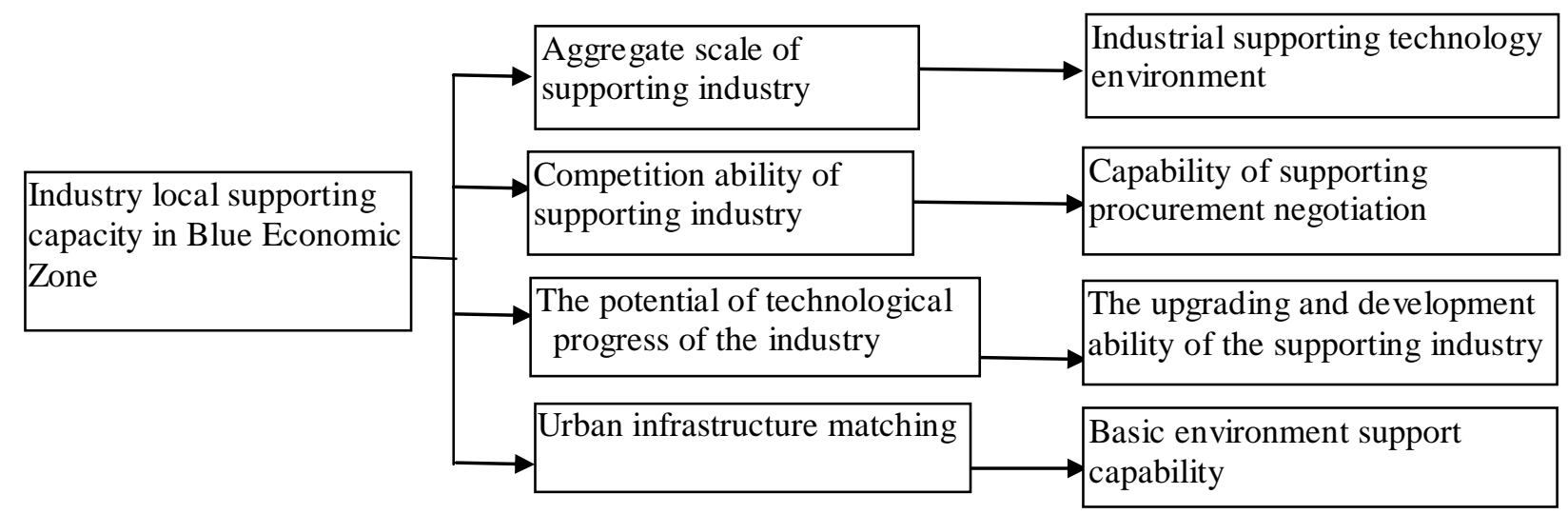

Figure 3. The index of the industry supporting capacity in the Blue Economic Industrial Zone

\section{Analysis of Industrial Matching in Qingdao Blue Economic Zone}

Analysis of Marine Biological Industry. Qingdao's marine bio medicine industry began in the 70s of last century, and has become one of the most important emerging industries in Qingdao. The marine bio pharmaceutical industry has also basically formed a complete industrial chain. It has basically established a marine biological industry system based on innovative drugs, biomedical materials, functional food and biological agricultural products. In the blue biological 
pharmaceutical industry park, there is the largest biological medicine professional incubator of China ${ }^{[8]}$.

The industrial development has a certain scale, but the level is not high. It is mainly composed of marine innovation, marine biological medical materials, marine functional food, marine biological and agricultural products and so on ${ }^{[10]}$. The scale of medical and biological products is generally small, the leading role of the faucet is not outstanding; the oceanic academic atmosphere is strong, and the conversion rate of scientific and technological achievements needs to be improved. The scientific and technological achievements in the field of marine industry in Qingdao are the first in the country, but the conversion rate is less than $1 / 10$.

The marine biological industry has the advantages of development, and the lower level of development. It is a lacking that the public service platform of research and development of marine medicine and biological products, pilot and industrialization. The platform of drug pharmacodynamic screening, drug safety assessment, clinical trials, animal experiments is almost blank.

Analysis of the Supporting Industry in the Sea Water Utilization. Seawater utilization includes three sub industries: direct sea water use, seawater desalination and other sea water utilization. At present, the key technologies of anticorrosion and antibiologic adhesion have broken the monopoly of overseas seawater desalination core technology. Have strong R \& D strength and good basic conditions. The main problem is lack of seawater desalination equipment manufacturing and internationally competitive specialized leading enterprises. The main problem is lack of seawater desalination equipment manufacturing and internationally competitive specialized leading enterprises.

Analysis of Marine Equipment Manufacturing Industry. Through the introduction of key project investment, Qingdao promotes the development of localization of marine equipment manufacturing industry, and forms a leading role in key supporting and key technology. At present, Qingdao is focusing on developing marine engineering equipment, ship repair and ship matching equipment, new energy equipment, environmental protection equipment, port machinery, power engineering equipment, petrochemical equipment and other large modern equipment manufacturing industry and ship parts manufacturing industry ${ }^{[2],[5]}$. It has been "made in Qingdao" on core components such as marine crankshaft and diesel engine. Qingdao has become one of the most perfect high-end shipbuilding bases in China. The problem is that the industrial categories are not homogeneous, the inter industry correlation is low, and the supporting capacity is not strong.

Analysis of Marine Transportation Logistics Industry. Qingdao port has been put into the country's largest container terminal, oil wharf, ore dock, world-class coal dock and bulk grain dock. There are trade relations with more than 450 ports in more than 130 countries and regions in the world. It has been formed that the multi-channel logistics transportation system with port as the hub by sea, railway, highway, air and pipeline. Through promoting the construction of professional functions of the port logistics, land and port logistics, Airport Logistics Park, to achieve the optimal layout of the regional and urban joint distribution network. Construction of three level distribution network system supported by logistics center, distribution center and terminal distribution network. Improve the construction of logistics node service system, and further optimize the construction of logistics facilities network. The main problems are inadequate multimodal transport coverage and matching, lack of comprehensive supporting functions of port, lack of leading enterprises with leading role, and coordinated development of supporting small and medium-sized enterprises.

\section{Countermeasures to Improve the Supporting Capacity of Industry}

Strengthening the Advantage Industry Chain and the Development of its Supporting Industry. The industrial chain of Qingdao's dominant marine industry will be extended and upgraded, and core supporting planning and construction are strengthened.

The port and shipping industry will fully build the international shipping hub port and international logistics center of Northeast Asia. The development model of Qingdao port has been changed from the expansion of quantity to the improvement of quality and efficiency. The port 
industry is transformed from the traditional loading and unloading industry to the modern logistics industry.

The development of marine biological medicine make full use of the advantageous geographical conditions for developing marine biological medicine industry in Qingdao, enrich the advantages of marine biological resources and strong marine biological research strength. Through the construction of the marine biotechnology innovation platform, to formulate special plans for the research and development of marine biology and medicine, we should promote the construction of the industrial base of marine bio medicine.

The marine engineering equipment industry will focus on the development of universal marine equipment, new marine equipment, forward-looking offshore equipment, key supporting equipment and systems. Through the improvement of the industrial chain, the marine engineering equipment industry cluster is built. We should improve the R \& D capability and create the development of industrial integration.

The comprehensive utilization of sea water industry is closely related to the core areas of the seawater desalination equipment industry. The manufacturing industry of seawater desalination equipment is developed by the leading equipment production integrated production enterprises. The series of low-temperature multi effect seawater desalination equipment, the localization of the reverse osmosis device. The production base of the desalination equipment in the north of China is built.

Marine new energy industry has a strong technical foundation and rich marine energy resources in Qingdao. Setting up a special fund for marine new energy in the Special funds for the blue economic zone of Shandong and Qingdao, to promote the development of related technology research and industrialization demonstration projects.

Countermeasures to Improve the Supporting Capacity of Local Industry. We should focus on the comprehensive efficiency and competitiveness of the key industry, and use the unbalanced and asymmetric matching strategy correctly. The emphasis is on industrial clusters or industrial chains, instead of individual enterprises taking industrial measures. We focus on the industry of the most promising and best suited for local development.

Reshaping the leading industry chain advantages, we focus on the key elements that can change the position of the enterprise in the global industry chain and the upgrading of the industry chain, instead of pursuing the integrity of the industrial chain unilaterally.

Supporting the development of the blue economy key development field industry matching. We pay attention to the important role of the key link construction for the whole industrial chain.

Making great efforts to support private small and medium-sized enterprises to participate in the construction of supporting industries, Concentrate on doing some deep part and adding "invisible champion". We will establish a win-win relationship between the leading enterprises and the small and medium-sized supporting enterprises, and promote the transformation and upgrading of the industrial structure of the Blue Economic Zone in Qingdao

Establish a "industry sharing service platform" to effectively aggregate industrial related factors such as talent, technology, market, resources, information, capital, logistics and so on. Guarantee the effective combination of "industry, universities, research institutions, capital and government" , to form the symbiotic ecological circle of all kinds of key elements in the industry. System integration of key elements of industry development, it lays a good foundation for the development of the whole industry.

\section{Measures to Improve the Supporting Capacity of the Industry}

We will formulate "Blue Economic Zone industry supporting suggestions" of across the region, and issue the catalogues and plans of the industry supporting, and build a relatively complete industrial chain from R \& D to core components manufacturing, machine assembly, logistics and after-sales service.

Promoting the construction of innovative Alliance of "production, learning and research". Relying on the National Key Laboratory, marine Science and technology base, regional aquaculture center, cooperation in the key technologies, core technologies and common technologies of marine 
emerging industries is jointly carried out.

We should seize the innovation opportunity given by the state to the first trial of the Blue Economic Zone in the financial field, and cultivate the financial service system and mechanism with the characteristics of the Blue Economic Zone, so as to give play to the guidance and promotion role of the industrial fund in the blue economic zone.

We should optimize the soft environment to enhance the matching rate of local industries, and explore the establishment of an industry supporting information network and integrated service platform with diversified investment, service specialization, market operation and socialization. Promote the effective integration and optimal allocation of innovative resources, such as talent, capital and technology.

Relying on the innovative service platform of marine advantageous industries and supporting industries in the blue economic zone of Qingdao, the soft environment for improving the local industrial matching rate in the blue economic zone of Qingdao is improved in an all-round way.

\section{Acknowledgements}

2015 major project of "The two region of the sea, the Yellow River" of Shandong province (No. 2015-63)

\section{Reference}

[1] Jun Ma. The optimization analysis of the marine industrial structure in the blue economic zone of Shandong Peninsula [J]. Economic Perspective (1), 2012(04):12-13.

[2] Qingdao Municipal Development and Reform Commission. Qingdao Blue Economic Zone Development Plan [Z].2012

[3] Baode Wang, Huixun Li. Study on the construction of cultural industry in Blue Economic Zone [J]. Shandong Social Sciences, 2012 (1): 9-12

[4] Haiping Zheng. Regional industry supporting research [J]. Research on productivity, 2004 (10): 126-128.

[5] Cheng Liu. Qingdao:the marine industry becomes a powerful engine of economy [N]. economic daily, 2017-3-26.

[6] Shucheng Han, Jing Li. Research on the mechanism of industrial transfer to enhance the supporting capacity of local enterprises [J]. China collective economy, 2010 (11): 33-34.

[7] Chunliang Gao. Transfer of International Manufacturing Optimal Industrial Matching and Policy [J]. China Industrial Economy, 2005(2):29-36.

[8] Chunli Wang,Yanxiong Zhu,Huan He. Research on the Development of Marine Bio Pharmaceutical Industry and Suggestions for the Development of Qingdao[EB/OL].http:// www.chinainfo.gov.cn,2014-03-04.

[9] Zhaoyuan Xu,Cui Hu,Youwe Lai. Industrial Supporting Capabilities and Productivity [J]. Research on Economics and Management, 2014,(7):74-84.

[10]Qingdao Municipal Development and Reform Commission. The development plan of Qingdao marine medicine and biological products industry (2013-2020)[R].2013.04. 